Supplement of Geosci. Model Dev., 8, 1763-1773, 2015

http://www.geosci-model-dev.net/8/1763/2015/

doi:10.5194/gmd-8-1763-2015-supplement

(C) Author(s) 2015. CC Attribution 3.0 License.

(c) (i)

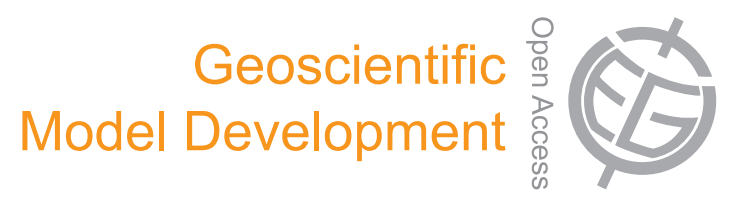

Supplement of

\title{
Path-integral method for the source apportionment of photochemical pollutants
}

\author{
A. M. Dunker \\ Correspondence to: A. M. Dunker (amdunker@gmail.com)
}

The copyright of individual parts of the supplement might differ from the CC-BY 3.0 licence. 


\section{Special Case - Taylor Series Expansions}

If there are two sources, the Taylor series expansions of the first-order sensitivities about the point $\Lambda=1$ (base case) through first order in $\lambda_{1}, \lambda_{2}$ are:

$$
\begin{gathered}
\frac{\partial c_{i}}{\partial \lambda_{1}}=\left.\frac{\partial c_{i}}{\partial \lambda_{1}}\right|_{\Lambda=1}+\left.\frac{\partial^{2} c_{i}}{\partial \lambda_{1}^{2}}\right|_{\Lambda=1}\left(\lambda_{1}-1\right)+\left.\frac{\partial^{2} c_{i}}{\partial \lambda_{1} \partial \lambda_{2}}\right|_{\Lambda=1}\left(\lambda_{2}-1\right) \\
\frac{\partial c_{i}}{\partial \lambda_{2}}=\left.\frac{\partial c_{i}}{\partial \lambda_{2}}\right|_{\Lambda=1}+\left.\frac{\partial^{2} c_{i}}{\partial \lambda_{2}^{2}}\right|_{\Lambda=1}\left(\lambda_{2}-1\right)+\left.\frac{\partial^{2} c_{i}}{\partial \lambda_{1} \partial \lambda_{2}}\right|_{\Lambda=1}\left(\lambda_{1}-1\right)
\end{gathered}
$$

Along the diagonal path, $\lambda_{1}=\lambda_{2}=s$. Substituting Eq. (S1) into Eq. (2) and integrating with respect to $s$ yields

$$
\begin{aligned}
S_{i 1}(\text { diag }) & =\left.\frac{\partial c_{i}}{\partial \lambda_{1}}\right|_{\Lambda=1}+\left.\left.\frac{\partial^{2} c_{i}}{\partial \lambda_{1}^{2}}\right|_{\Lambda=1} \frac{(s-1)^{2}}{2}\right|_{0} ^{1}+\left.\left.\frac{\partial^{2} c_{i}}{\partial \lambda_{1} \partial \lambda_{2}}\right|_{\Lambda=1} \frac{(s-1)^{2}}{2}\right|_{0} ^{1} \\
& =\left.\frac{\partial c_{i}}{\partial \lambda_{1}}\right|_{\Lambda=1}-\frac{1}{2}\left(\left.\frac{\partial^{2} c_{i}}{\partial \lambda_{1}^{2}}\right|_{\Lambda=1}+\left.\frac{\partial^{2} c_{i}}{\partial \lambda_{1} \partial \lambda_{2}}\right|_{\Lambda=1}\right)
\end{aligned}
$$

Similarly,

$$
S_{i 2}(\text { diag })=\left.\frac{\partial c_{i}}{\partial \lambda_{2}}\right|_{\Lambda=1}-\frac{1}{2}\left(\left.\frac{\partial^{2} c_{i}}{\partial \lambda_{2}{ }^{2}}\right|_{\Lambda=1}+\left.\frac{\partial^{2} c_{i}}{\partial \lambda_{1} \partial \lambda_{2}}\right|_{\Lambda=1}\right)
$$

For the path $B-b 1-b$ in Fig. $1, \lambda_{2}=0$ from $b$ to $b 1$, and $d \lambda_{1}=0$ from $b 1$ to $B$. Using this information and integrating Eq. (S1) with respect to $\lambda_{1}$ from $b$ to $b 1$ gives

$$
\begin{aligned}
S_{i 1}(B-b 1-b) & =\left.\frac{\partial c_{i}}{\partial \lambda_{1}}\right|_{\Lambda=1}+\left.\left.\frac{\partial^{2} c_{i}}{\partial \lambda_{1}^{2}}\right|_{\Lambda=1} \frac{\left(\lambda_{1}-1\right)^{2}}{2}\right|_{0} ^{1}-\left.\frac{\partial^{2} c_{i}}{\partial \lambda_{1} \partial \lambda_{2}}\right|_{\Lambda=1} \\
& =\left.\frac{\partial c_{i}}{\partial \lambda_{1}}\right|_{\Lambda=1}-\left.\frac{1}{2} \frac{\partial^{2} c_{i}}{\partial \lambda_{1}{ }^{2}}\right|_{\Lambda=1}-\left.\frac{\partial^{2} c_{i}}{\partial \lambda_{1} \partial \lambda_{2}}\right|_{\Lambda=1}
\end{aligned}
$$

Noting that $d \lambda_{2}=0$ from $b$ to $b 1$ and that $\lambda_{1}=1$ from $b 1$ to $B$ and integrating Eq. (S2) with respect to $\lambda_{2}$ from $b 1$ to $B$ gives

$$
S_{i 2}(B-b 1-b)=\left.\frac{\partial c_{i}}{\partial \lambda_{2}}\right|_{\Lambda=1}-\left.\frac{1}{2} \frac{\partial^{2} c_{i}}{\partial \lambda_{2}{ }^{2}}\right|_{\Lambda=1}
$$

By a procedure analogous to that for path $B-b 1-b$, the results for path $B-b 2-b$ are:

$$
\begin{aligned}
& S_{i 1}(B-b 2-b)=\left.\frac{\partial c_{i}}{\partial \lambda_{1}}\right|_{\Lambda=1}-\left.\frac{1}{2} \frac{\partial^{2} c_{i}}{\partial \lambda_{1}{ }^{2}}\right|_{\Lambda=1} \\
& S_{i 2}(B-b 2-b)=\left.\frac{\partial c_{i}}{\partial \lambda_{2}}\right|_{\Lambda=1}-\left.\frac{1}{2} \frac{\partial^{2} c_{i}}{\partial \lambda_{2}{ }^{2}}\right|_{\Lambda=1}-\left.\frac{\partial^{2} c_{i}}{\partial \lambda_{1} \partial \lambda_{2}}\right|_{\Lambda=1}
\end{aligned}
$$

The expansion of the concentration in a Taylor series about $\Lambda=1$ through second order is: 


$$
\begin{gathered}
c_{i}(\boldsymbol{\Lambda})=c_{i}(\Lambda=1)+\left.\frac{\partial c_{i}}{\partial \lambda_{1}}\right|_{\Lambda=1}\left(\lambda_{1}-1\right)+\left.\frac{\partial c_{i}}{\partial \lambda_{2}}\right|_{\Lambda=1}\left(\lambda_{2}-1\right)+\left.\frac{1}{2} \frac{\partial^{2} c_{i}}{\partial \lambda_{1}^{2}}\right|_{\Lambda=1}\left(\lambda_{1}-1\right)^{2} \\
+\left.\frac{1}{2} \frac{\partial^{2} c_{i}}{\partial \lambda_{2}^{2}}\right|_{\Lambda=1}\left(\lambda_{2}-1\right)^{2}+\left.\frac{\partial^{2} c_{i}}{\partial \lambda_{1} \partial \lambda_{2}}\right|_{\Lambda=1}\left(\lambda_{1}-1\right)\left(\lambda_{2}-1\right)
\end{gathered}
$$

With this expansion, the concentration difference $\Delta c_{i}=c_{i}(\Lambda=1)-c_{i}(\Lambda=0)$ is then

$$
\Delta c_{i}=\left.\frac{\partial c_{i}}{\partial \lambda_{1}}\right|_{\Lambda=1}+\left.\frac{\partial c_{i}}{\partial \lambda_{2}}\right|_{\Lambda=1}-\left.\frac{1}{2} \frac{\partial^{2} c_{i}}{\partial \lambda_{1}^{2}}\right|_{\Lambda=1}-\left.\frac{1}{2} \frac{\partial^{2} c_{i}}{\partial \lambda_{2}{ }^{2}}\right|_{\Lambda=1}-\left.\frac{\partial^{2} c_{i}}{\partial \lambda_{1} \partial \lambda_{2}}\right|_{\Lambda=1}
$$

For all three paths, $\Delta c_{i}=S_{i 1}+S_{i 2}$, and thus the source apportionments equal the concentration difference between the base and background cases through second-order in $\lambda_{1}$ and $\lambda_{2}$. 
Table S1. Input data for the CAMx simulations.

\begin{tabular}{|c|c|c|}
\hline Latitude & N $34^{\circ}$ (Los Angeles/Atlanta) & \\
\hline Days & June $20-22$ & \\
\hline Temperature $(\mathrm{K})$, diurnally varying & Cell 1: $290 \rightarrow 305 \rightarrow 290$ & Cell 2: $295 \rightarrow 307 \rightarrow 295$ \\
\hline Cell height $(\mathrm{m})$, diurnally varying & Cell 1: $100 \rightarrow 300 \rightarrow 100$ & Cell 2: $1400 \rightarrow 1200 \rightarrow 1400$ \\
\hline Deposition & Wesely/Slinn algorithm ${ }^{a, b}$ & \\
\hline Chemistry & Carbon Bond 6 & \\
\hline Initial VOC (ppbC $\left.{ }^{c}\right)$ & 8.0 & \\
\hline Initial VOC/NOx (ppbC/ppb) & 16. & \\
\hline Species & Description & Initial Concentration $(p p b)^{d}$ \\
\hline $\mathrm{O}_{3}$ & & 35. \\
\hline NO & & 0.1 \\
\hline $\mathrm{NO}_{2}$ & & 0.4 \\
\hline $\mathrm{CO}$ & & 120. \\
\hline $\mathrm{SO}_{2}$ & & 1. \\
\hline ACET & acetone & 0.0 \\
\hline ALD2 & acetaldehyde & 0.05 \\
\hline ALDX & C3 \& higher aldehydes & 0.01 \\
\hline BENZ & benzene & 0.0 \\
\hline ETH & ethene & 0.11 \\
\hline ETHA & ethane & 0.02 \\
\hline ETHY & ethyne & 0.0 \\
\hline $\mathrm{ETOH}$ & ethanol & 0.11 \\
\hline FORM & formaldehyde & 0.10 \\
\hline IOLE & internal olefin carbon & 0.14 \\
\hline ISOP & isoprene & 0.57 \\
\hline KET & ketone carbon & 0.0 \\
\hline $\mathrm{MEOH}$ & methanol & 0.33 \\
\hline OLE & terminal olefin carbon & 0.21 \\
\hline PAR & paraffin carbon & 1.25 \\
\hline PRPA & propane & 0.0 \\
\hline TERP & mono-terpenes & 0.16 \\
\hline TOL & mono-alkyl aromatics & 0.0 \\
\hline$X Y L$ & poly-alkyl aromatics & 0.0 \\
\hline NR & non-reactive carbon & 0.27 \\
\hline
\end{tabular}

${ }^{a}$ Wesely, M.L.: Parameterization of surface resistances to gaseous dry deposition in regional-scale numerical models, Atmos. Environ., 23, 1293-1304, 1989.

b Slinn, S.A., and Slinn, W.G.N.: Predictions for particle deposition on natural waters, Atmos. Environ., 24, 1013-1016, 1980.

c Parts per billion of carbon

d Initial concentrations of the VOC species are in the same relative proportions as the daily biogenic emissions. 
Table S2. All daily emission rates used in the base-case simulation.

\begin{tabular}{lcccccc}
\hline Species & \multicolumn{5}{c}{ Emission Rate $\left(\mathrm{mol} \mathrm{day}^{-1} \mathrm{~km}^{-2}\right)$} \\
\cline { 2 - 6 } & $\begin{array}{c}\text { Biogenic } \\
\text { Sources }^{\mathrm{a}}\end{array}$ & $\begin{array}{c}\text { Fuel } \\
\text { Combustion }\end{array}$ & $\begin{array}{c}\text { Industrial } \\
\text { Sources }\end{array}$ & $\begin{array}{c}\text { On-road } \\
\text { Vehicles }\end{array}$ & $\begin{array}{c}\text { Non-road } \\
\text { Vehicles }\end{array}$ & $\begin{array}{c}\text { Other } \\
\text { Sources }\end{array}$ \\
\hline NO & 13.5 & 77.4 & 19.7 & 132.9 & 73.2 & 1.9 \\
NO2 & 0.00 & 8.60 & 2.19 & 13.59 & 7.48 & 0.21 \\
HONO & 0.00 & 0.00 & 0.00 & 1.18 & 0.65 & 0.00 \\
CO & 35.9 & 51.8 & 58.2 & 1158.4 & 683.0 & 57.0 \\
ACET & 0.00 & 0.00 & 10.54 & 0.11 & 0.06 & 0.00 \\
ALD2 & 2.39 & 0.00 & 0.03 & 0.69 & 0.71 & 0.00 \\
ALDX & 0.61 & 0.00 & 0.62 & 0.10 & 0.22 & 0.01 \\
BENZ & 0.00 & 0.14 & 0.10 & 1.47 & 1.66 & 0.04 \\
ETH & 5.38 & 0.00 & 0.03 & 6.72 & 4.85 & 0.00 \\
ETHA & 1.21 & 0.00 & 5.90 & 1.69 & 1.77 & 0.00 \\
ETHY & 0.00 & 0.00 & 0.00 & 1.19 & 2.87 & 0.00 \\
ETOH & 5.38 & 0.00 & 1.23 & 6.16 & 2.61 & 2.78 \\
FORM & 4.78 & 0.73 & 0.25 & 1.52 & 1.73 & 0.13 \\
IOLE & 7.07 & 0.00 & 0.06 & 1.12 & 0.66 & 0.38 \\
ISOP & 28.82 & 0.00 & 0.00 & 0.04 & 0.00 & 0.00 \\
KET & 0.00 & 0.00 & 1.00 & 0.00 & 0.03 & 0.00 \\
MEOH & 16.47 & 0.00 & 4.15 & 0.37 & 0.00 & 0.00 \\
OLE & 10.32 & 0.00 & 0.77 & 3.40 & 6.27 & 0.36 \\
PAR & 62.61 & 4.95 & 189.04 & 96.74 & 82.80 & 54.34 \\
PRPA & 0.00 & 0.25 & 13.63 & 0.17 & 0.21 & 0.01 \\
TERP & 8.17 & 0.00 & 0.38 & 0.00 & 0.00 & 0.00 \\
TOL & 0.00 & 0.06 & 5.91 & 4.48 & 4.21 & 0.43 \\
XYL & 0.00 & 0.00 & 3.15 & 2.69 & 3.45 & 0.13 \\
NR & 13.55 & 0.01 & 7.50 & 1.19 & 0.99 & 0.70 \\
\hline
\end{tabular}

ancludes lightning 
Table S3. Average error and bias for different numerical integration formulas. The sum of the source contributions calculated using the formula is compared to the anthropogenic increment of $\mathrm{NO}_{2}$ or $\mathrm{HNO}_{3}$.

\begin{tabular}{|c|c|c|c|}
\hline Path & Formula $^{a}$ & $\begin{array}{l}\text { Mean Absolute Error } \\
(\mathrm{ppb})\end{array}$ & $\begin{array}{c}\text { Mean Bias } \\
(\mathrm{ppb})\end{array}$ \\
\hline \multicolumn{4}{|c|}{$\mathrm{NO}_{2}$ Increment } \\
\hline Diag & TR2 & 2.74 & 2.66 \\
\hline Diag & GL2s & 0.19 & -0.15 \\
\hline Diag & $\mathrm{GL} 2 r$ & 0.49 & -0.45 \\
\hline Diag & GL3s & 0.06 & -0.05 \\
\hline Diag & GL3r & 0.13 & -0.08 \\
\hline Diag & GL4s & 0.04 & -0.03 \\
\hline Diag & GL4r & 0.07 & -0.01 \\
\hline NOxF & GL3s & 0.20 & 0.00 \\
\hline NOxF & GL3r & 1.12 & -1.06 \\
\hline NOxF & GL4s & 0.12 & -0.04 \\
\hline VOCF & GL3s & 1.49 & 1.46 \\
\hline VOCF & GL3r & 3.06 & -3.05 \\
\hline VOCF & GL4s & 0.76 & -0.73 \\
\hline \multicolumn{4}{|c|}{$\mathrm{HNO}_{3}$ Increment } \\
\hline Diag & TR2 & 4.55 & -4.55 \\
\hline Diag & GL2s & 0.28 & 0.06 \\
\hline Diag & GL2r & 0.53 & -0.15 \\
\hline Diag & GL3s & 0.20 & -0.18 \\
\hline Diag & GL3r & 0.24 & -0.19 \\
\hline Diag & GL4s & 0.28 & -0.27 \\
\hline Diag & GL4r & 0.28 & -0.28 \\
\hline NOxF & GL3s & 0.44 & 0.40 \\
\hline NOxF & GL3r & 0.80 & -0.35 \\
\hline NOxF & GL4s & 0.57 & -0.57 \\
\hline VOCF & GL3s & 1.37 & -1.32 \\
\hline VOCF & GL3r & 1.99 & 1.97 \\
\hline VOCF & GL4s & 0.54 & 0.47 \\
\hline
\end{tabular}

${ }_{\mathrm{a}}^{\mathrm{TR} 2}=$ trapezoidal rule, 2 points. GLnx = Gauss-Legendre formula using $n$ points and $x$ as the integration variable.

${ }^{\mathrm{b}}$ Hourly average over the 3-day simulation. 

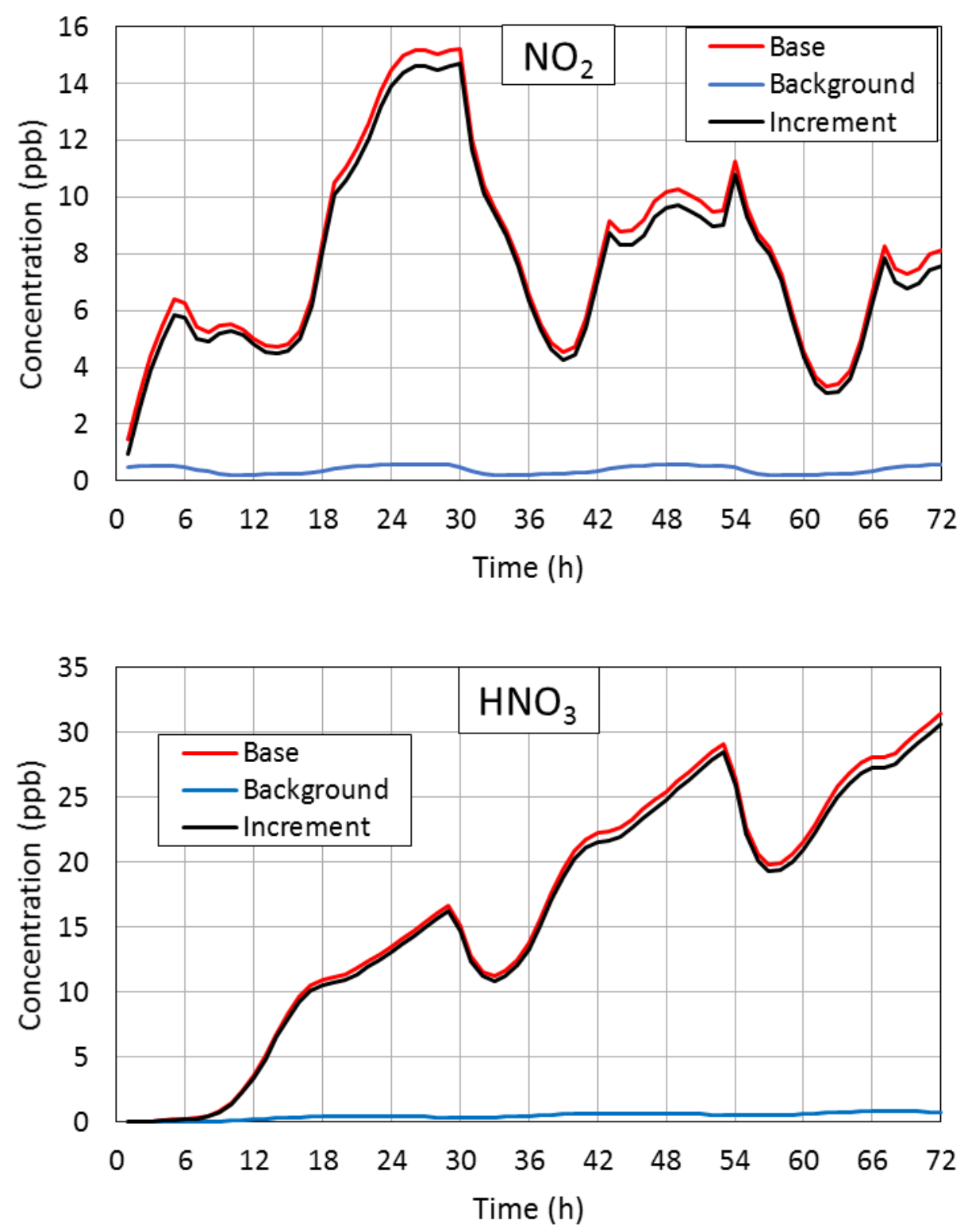

Figure S1. Concentrations of $\mathrm{NO}_{2}$ and $\mathrm{HNO}_{3}$ from the base case, the background case and the anthropogenic increment. 

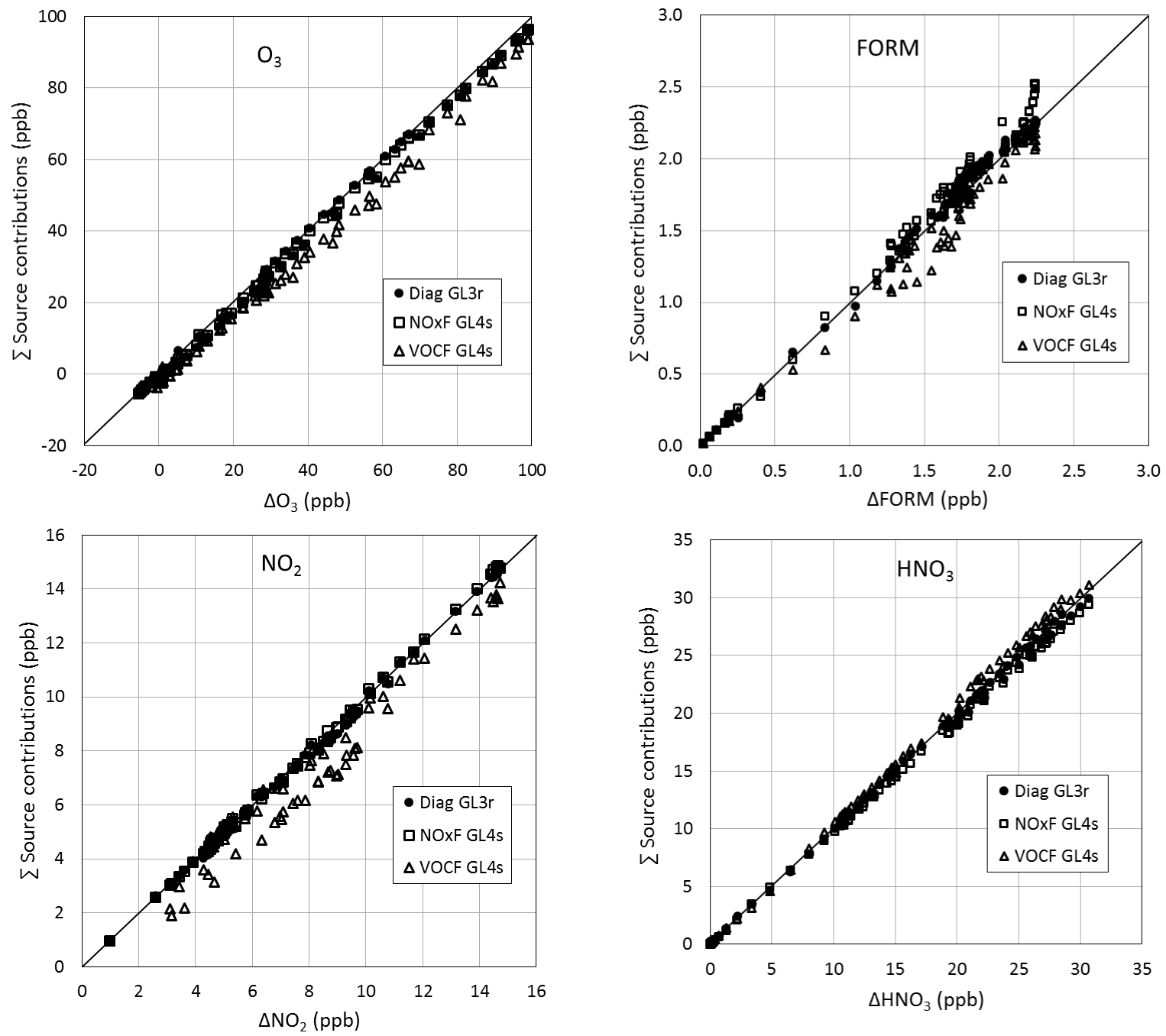

Figure S2. Comparison of the sum of the source contributions to the anthropogenic increment. Points are hourly values during the 3-day simulation. Also shown is a 1-to-1 correspondence line. 

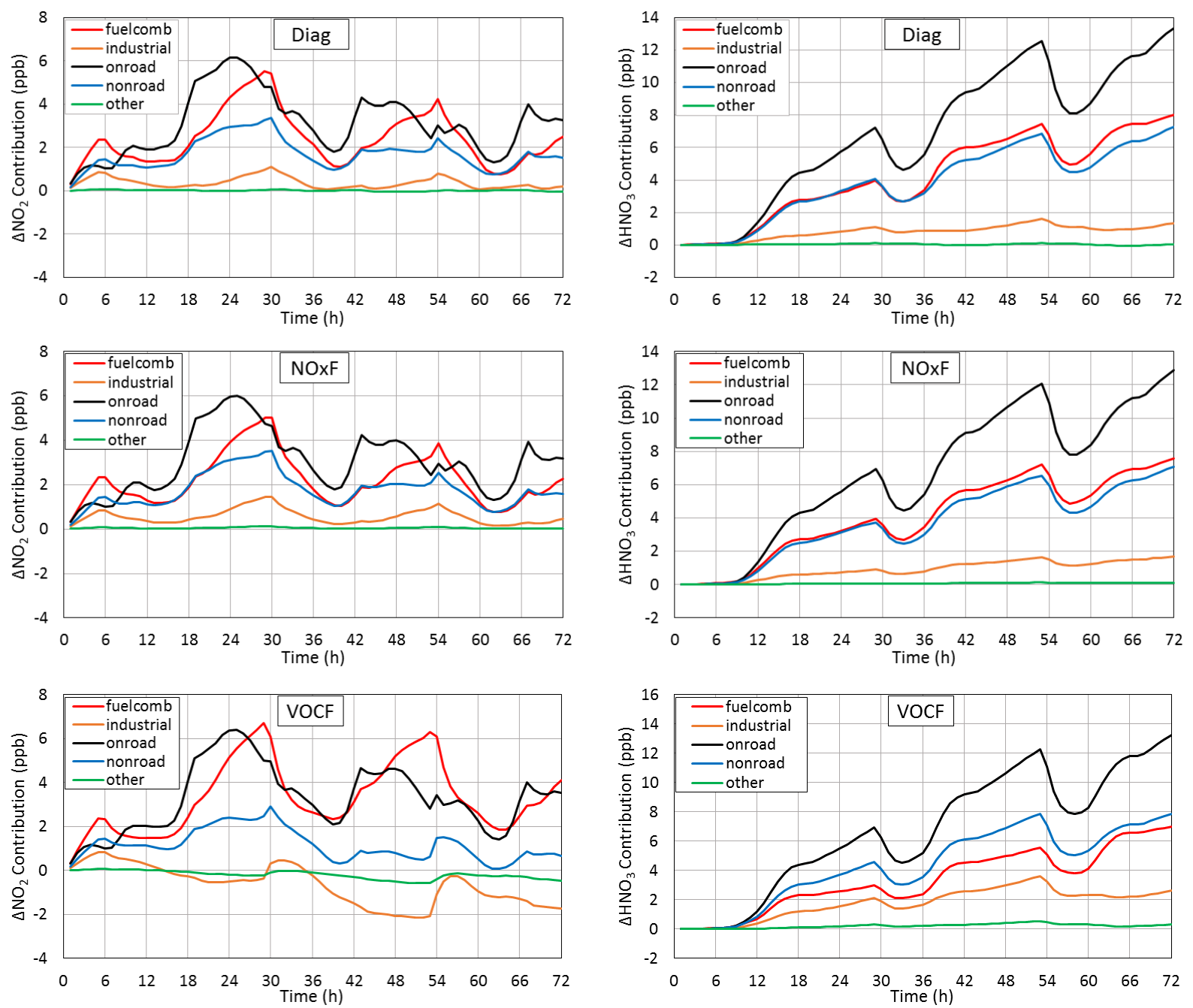

Figure S3. Apportionment of the anthropogenic $\mathrm{NO}_{2}$ increment (left) and $\mathrm{HNO}_{3}$ increment (right) to sources using the Diag, NOxF, and VOCF emission-control paths. 